This item was submitted to Loughborough's Research Repository by the author.

Items in Figshare are protected by copyright, with all rights reserved, unless otherwise indicated.

\title{
Additively manufactured flow-resistive pulse sensors
}

\section{PLEASE CITE THE PUBLISHED VERSION}

https://doi.org/10.1021/acs.analchem.8b05140

\section{PUBLISHER}

(c) American Chemical Society (ACS)

\section{VERSION}

AM (Accepted Manuscript)

\section{PUBLISHER STATEMENT}

This document is the Accepted Manuscript version of a Published Work that appeared in final form in Analytical Chemistry, copyright ( $)$ American Chemical Society after peer review and technical editing by the publisher. To access the final edited and published work see https://pubs.acs.org/doi/10.1021/acs.analchem.8b05140.

\section{LICENCE}

CC BY-NC-ND 4.0

\section{REPOSITORY RECORD}

Hampson, Sarah, Marcus Pollard, Peter Hauer, Hayden Salway, Steven Christie, and Mark Platt. 2019. "Additively Manufactured Flow-resistive Pulse Sensors". figshare. https://hdl.handle.net/2134/36711. 


\section{Additively Manufactured Flow Resistive Pulse Sensors}

Sarah M. Hampson ${ }^{1}$, Marcus Pollard ${ }^{1}$, Peter Hauer ${ }^{2}$, Hayden Salway ${ }^{1}$, Steven D. R. Christie ${ }^{1}$ and Mark Platt ${ }^{+*}$

1. Department of Chemistry, Loughborough University, Loughborough, Leicestershire, LE11 3TU, UK.

2. School of Chemical and Physical Sciences, Victoria

University of Wellington, Wellington 6140, New Zealand

*m.platt@lboro.ac.uk 


\begin{abstract}
Resistive Pulse Sensors, RPS, provide detailed characterisation of materials from the nanoparticle up to large biological cells on a particle-to-particle basis. During the RPS experiment, particles pass through a channel or pore which conduct ions, and the change in ionic current versus time is monitored. The change in current during each translocation, also known as a 'pulse', is dependent upon the ratio of the particle and channel dimensions. Here we present a facile and rapid method for producing FlowRPS sensors which do not require lithographic processes. The Additively Manufactured, AM, sensor has channel dimensions that can be easily controlled. In addition, the fabrication process allows the sensor to be quickly assembled, disassembled, cleaned and reused. Further, the RPS sensor can be created with a direct interface for fluidic pumps or imaging window for complimentary optical microscopy. We present experiments and simulations of the RPS sensor, showing how the pulse shape are dependent upon the channel morphology and how the device can count and size particles across a range of flow rates and ionic strengths. The use of pressure-driven fluid flow through the device allowed a rapid characterisation of particles down to concentrations as low as $1 \times 10^{-3}$ particles per $\mathrm{ml}$ which equated to one event per second.
\end{abstract}

Key words: Additive Manufacturing, Resistive Pulse Sensor, Particle Counter, Flow. 
Interest in nanoscale channels within synthetic materials have grown over the last two decades. $^{1-3}$ These channels have applications in biosensing, ${ }^{4-6}$ materials characterisation $n^{7,8}$ and mimicking biological systems, enabling the study of ionic transport within confined geometries. ${ }^{9-12}$ The translocation of a nanoparticle or analyte through these channels, can be monitored by measuring the ionic current. Each translocation event causes a change to the conductance of the channel known as a Resistive Pulse, which is related to the physical properties of the analyte. Resistive Pulse Sensors, RPS, are highly attractive sensing platforms that have single particle/analyte resolution. Information on analyte size, concentration and charge can be measured quickly, reliably and to a high level of sensitivity. ${ }^{8,13-15}$ Indeed, RPS has exhibited high resolution for the monitoring various analytes, including DNA, ${ }^{6}$ proteins, ${ }^{4}$ and even whole cells. ${ }^{16}$

To date RPS have been created in a range of materials from graphene, ${ }^{17-19}$ polymers, ${ }^{20}$ silicon nitride ${ }^{21}$ and glass. ${ }^{9,22-25}$ Their sensitivity is directly related to the dimensions of the channel. The transport through the pore or channel is controlled by tuning the potential difference, charge on the pore wall, electrophoretic mobility of the analyte, supporting electrolyte concentration and induced convection. ${ }^{26,27}$ Maintaining sensitivity whilst retaining high count rates is a difficult challenge as the pulse frequency is directly related to the pore size. ${ }^{28}$ Recent advances in the RPS sensors have utilised pores of different aspect ratios and shapes or specific electrode configurations, to improve signal to noise ratio. ${ }^{29-31}$ As well as being easier to fabricate the use of larger pores has the added benefit that a higher flow rate of liquid through the pore can be maintained, reducing the time required to acquire the data set. It also prevents a major drawback of RPS sensors, namely the blocking of small pores. An emerging fabrication process for microfluidic systems is additive manufacturing or 3D printing. This has been suggested as an alternative approach to microfluidic fabrication, mainly due to its ability to build a complete part from an STL (standard tessellation language) file with no intermediate steps, thus minimising labour, time and costs. ${ }^{32}$ As such in the field of microfluidics the use of 3D printing and lab-on-a-chip fabrication has increased. ${ }^{33-36}$ An overview of microfluidic technologies made by each type of additive manufacturing is given by Folch, ${ }^{37}$ but SLA (stereolithography) has proven to be the most popular for this field, due to its superior resolution.

Here we present a facile method for producing an RPS sensor using additive manufacturing, AM. The channel diameter, height and length are controlled, and we demonstrate the ability of the RPS to count particles across a range of lonic strengths $(1-50 \mathrm{mM} \mathrm{KCl})$ which have biological, environment and manufacturing applications. The ability to print the sensor with integrated screw threads into the device allows it to be connected to flow systems. The use of flow allowed particles to be counted quickly and recorded an event per second at low particle concentrations, $1 \times 10^{-3}$ particles $/ \mathrm{ml}$. We also present result about the baseline and pulse shape obtained with the Finite Element Method (FEM) and illustrate how the pulses reveal information about the channel shape.

\section{Experimental Section}




\section{Device Printing}

Design CAD drafts were drawn and converted to .STL format on Siemens NX Version 11.0 software. Pore devices were printed in Accura $₫ 60$ polymer on a Viper si2 SLA printer (both 3D Systems) in the pre-set high resolution (HR) build mode, which uses

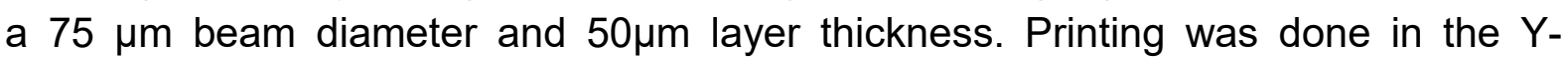
direction. After printing, any uncured resin was removed by rinsing the devices with IPA (VWR Chemicals) and methanol (VWR chemicals).

\section{Device Assembly}

Silver wire electrodes $(0.25 \mathrm{~mm}$ diameter, purity $99.99 \%$, obtained from Advent Research Materials, cat. No. AG5485) were first polished by hand on a polishing pad (800 Grit, obtained from BASi), before alignment was carried out by the use of grooves for electrode housing in the CAD file: device channels and electrode grooves were printed open, and silver wires were pressed into the grooves and aligned with the microchannel walls by hand, under a microscope. The devices were sealed with TESA 4965 double-sided tape (obtained from 3M) and LEXANTM 8010PC polycarbonate film (obtained from SABIC, $250 \mu \mathrm{m}$ thickness), applied by hand and flattened with a rubber nub. The electrodes were then sealed in place with ARALDITE® RAPID epoxy adhesive to ensure the electrodes did not move during operation.

\section{Device Images}

Device images were taken on an Optiphot-2 optical microscope using a DS-5M camera and a DS-L1 Camera Control Unit (all obtained from Nikon), with the exception of the pore channel photographs, which were taken on a DM2500 microscope (obtained from Leica). Pore channels were measured via Image-J (Version 1.5, NIH). Device SEM images were taken on a TM3030 benchtop scanning electron microscope (Hitachi).

\section{Data Acquisition Set-up}

Microfluidic flow was driven by Mitos P-Pump Basic pressure- driven pumps using Flow Control Centre V3.1.44 software (both Dolomite) and fed into the device by standard HPLC fittings in printed threads. Electrical noise shielding was done by the use of a custom-built Faraday cage (steel, $1 \mathrm{~mm}$ thick, obtained from Nanopore Solutions). Electronic measurement and control were carried out via a QSensing3 current amplifier (Izon Science), controlled by Izon Control Suite Software Version V3.1.2.53. Data was obtained using a $15 \mathrm{kHz}$ filter, $50 \mathrm{kHz}$ sampling frequency, low gain mode. The default minimum blockade height $(0.05 \mathrm{nA})$ for particle detection was used.

\section{ICR measurements}

Current measurements were recorded over a range of $\pm 300 \mathrm{mV}$ for each solution of $\mathrm{KCl}$ (obtained from Fischer Scientific), $\mathrm{KCl}$ solutions were prepared in deionised water to the following concentrations: $0.5 \mathrm{mM}, 1.0 \mathrm{mM}, 2.5 \mathrm{mM}$ and $10 \mathrm{mM}$, The current reading was taken after 10 seconds to allow for stabilisation. The flow rate experiments 
were recorded using $1 \mathrm{mM} \mathrm{KCl}$ solution, the flow rate was varied using the pumps and control software stated above.

\section{Particle counting and sizing}

Analysis was carried out on suspensions of polystyrene particles $(30 \mu \mathrm{m}, \mathrm{COOH}-$ coated, obtained from Sigma-Aldrich, cat. no. 84135, and $20 \mu \mathrm{m}$, micromer® $\mathrm{COOH}-$ coated, (obtained from Micromod Partikeltechnologie $\mathrm{GmbH}$, cat. no. 01-02-204) diluted in either $\mathrm{KCl}$ (obtained from Fischer Scientific) or PBS (obtained from Sigma Aldrich). Each particle suspension run was recorded for 60 s.

\section{Theory and Modelling}

The size and shape of the resistive pulse is mainly dependant on the dimensions and geometry of both the particle and the sensing region. The relative change of the current upon a particle translocation is in general related to the physical volumetric blocking of the electrolyte solution, which is often described as the underlying principle behind resistive pulse sensing. ${ }^{38}$ To calculate the baseline current and the resistive pulse in a RPS device the underlying electrostatic problem has to be solved. In a geometric domain with uniform, isotropic conductivity $\sigma$ and under the assumption that there are no current sources or sinks within the domain, the electric potential $\varphi$, is determined by the Laplace equation $\Delta \varphi=0$. With the knowledge of $\varphi$, the electric field $\vec{E}$, the current density $\vec{\jmath}$ can then be calculated via $\vec{E}=-\nabla \varphi$ and $\vec{\jmath}=\vec{\sigma} \vec{E}$. While for some specific geometries an analytic solution for the Laplace equation can be found, this is not the case for the geometries used in this work. The addition of a particle in the domain complicates the calculation further. It has to be mentioned that the charge of the particle and the channel have to be considered under certain circumstances to obtain the correct orientation and size of the pulse. ${ }^{39}$ However, charge effects are only relevant for much smaller structures than used here, which are in the order of $100 \mu \mathrm{m}$, and can therefore be assumed negligible.

There are several approaches that use approximations to calculate the resistive pulse. For example Deblois ${ }^{40}$ used an expression found by Maxwell to calculate the resistive pulse. In the simple case of a spherical particle in a cylindrical pore and introduced a correction term for particles that become comparable in size to the pore diameter. Gregg and Steidley's resistance approach ${ }^{41}$ has been used to find solutions for various geometries. Although its simplicity makes it easy to use and fast to calculate the so calculated resistance is only a lower bound and has, for the case of a sphere in a cylindrical pore (sphere diameter small compared to the cylinder), to be multiplied by a factor of 1.5 to agree with the correct value. ${ }^{40}$

For the geometry used here and the particular orientation of the electrodes, which are perpendicular and in very close proximity to the pore channel, the Finite Element Method $(\mathrm{FEM})^{42}$ is the most suitable approach for both calculating the baseline current and the resistive pulse. Due to the increase in computational power FEM has become an important tool to solve various physical problems found in RPS sensors governed by Partial Differential Equations (PDE). ${ }^{10,43,44} \mathrm{~A}$ typical workflow for an FEM simulation involves drawing the geometry (Figure S1a), assigning the material 
properties and defining the boundary conditions. Here the boundary condition for the channel walls and the particle, which can be assumed to be insulating, is $\overrightarrow{n \nabla} \varphi=0$, meaning that the component of the electric field normal to the boundary has to vanish at the surface. The potential at one electrode is set to V0 and at the other to 0 . Then a mesh (Figure S1b) is created and the simulation is executed. It was assured that the mesh resolution had no significant (less than $0.1 \%$ ) influence on the results. The baseline current $l_{0}$ through the pore is then extracted from the simulation by integrating the normal current density over one electrode. To obtain the resistive pulse the particle was placed at various positions close to and inside the sensing channel. The equations used in the model are outlined within the supplementary material.

\section{Results and discussion}

Creating small channels within AM devices can cause difficulties. After the print process the monomer, resin or powder left within the channels needs to be removed. For fluidic applications this residual material can result in channels being blocked. As the channels decrease in size, the difficulty in removing all the residue increases. To overcome this problem here we have utilised a similar approach to our AM optical counters, ${ }^{32}$ where smaller channels are printed onto the surface of the material, and then sealed with a polymer film. This allows us to produce an SLA printed device with a resolution of circa $75 \mu \mathrm{m}$. We acknowledge that other AM technologies can produce smaller channels. However, our current interest lies within larger constructs that contain connectors and mixing channels with total dimensions over $1 \mathrm{~cm}$. Figure 1ai and ii shows the CAD files for two variations of the RPS sensor. Both are designed to be integrated into a flow system and connected to pumps, and have printed screw threads, labelled inlet/ outlet. A benefit of the AM process is that the sensor and screw threads can be easily fabricated together in a configuration that matches the application/ chip holder.

ai)

aii)
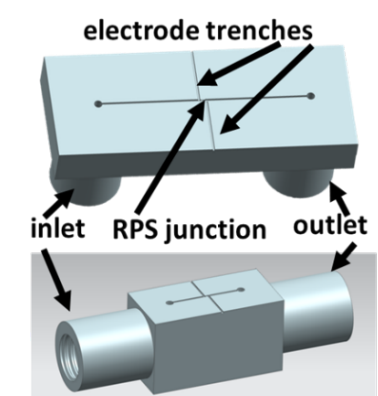

c)

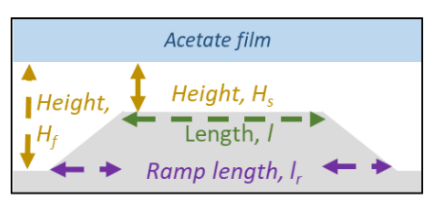

b)

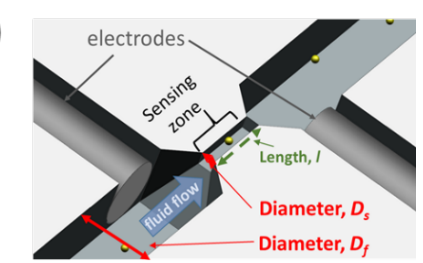

d)

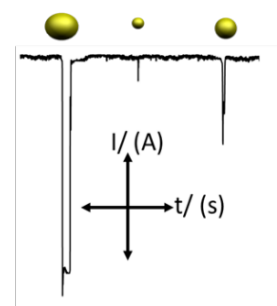

Figure 1a) CAD for two RPS sensors with different flow designs. b) Enlarged section of the sensing zone showing the electrodes and diameter of the sensing zone, $D_{s}$, and flow, $D_{f .}$. C) Cross section of the sensing zone, showing flow channel height, $H_{f}$, sensing zone height, $H_{s}$, 
Length, $I$, and ramp length $I_{r}$. d) Schematic of three resistive pulses from particles of different diameters.

The channel goes from the inlet up to the surface of the design, and then runs along the top surface forming a trench, figure $1 \mathrm{~b}$ shows a schematic. The centre of the flow channel narrows to form the sensing zone of the RPS device. The sensing zone is flanked by the two $\mathrm{Ag} / \mathrm{AgCl}$ electrodes. A magnified section of the design is shown in figures $1 \mathrm{~b}$ and $\mathrm{c}$. The channels are sealed by adhering a Polycarbonate (PC) film on top of the device as described above. ${ }^{32}$ The dimensions of the sensing zone are defined by its height, $H_{s}$, length, $I$, and diameter $D_{s}$. The main flow channel has a diameter and height, $D_{f}$ and $H_{f}$ respectively. To bridge the flow region to the sensing zone we placed a ramp rather than a shear face. The ramp changed the height of the channel over a distance $I_{r}$ and was the same at the entrance and exit of the sensing region. When a particle passes through the electrolyte-filled channel, under the application of a potential difference between the two electrodes, each particle is recorded as a resistive pulse. The pulse size and shape are dependent upon its the channel and pore dimensions, as shown in figure $1 \mathrm{~d}$, and can be calculated as described above.

Optical and electron microscope images of an RPS devices are shown in figure $2 a$ and $b$ respectively. In the optical image the location and alignment of the electrodes can be clearly seen. The SEM image in figure $2 b$ shows the ramp and surface roughness on a typical printed device. Figure $2 c$ shows examples of the RPS where $D_{s}$ is systematically decreased from $110 \mu \mathrm{m}$ to $40 \mu \mathrm{m}$. It was found across multiple attempts that as the intended channel diameter approached $40 \mu \mathrm{m}$, the AM process failed to produce a clear feature. Figure S2 shows the relationship between intended and recorded $D_{s}$, measured at the pore mouth within each channel and across 6 replicate parts. Figure S2 also reports the variation in channel with across the build. This is taken from 15 measurements down the length of the channel. It should be noted that in the designs used here, $D_{s}$ always equals $\mathrm{H}_{s}$, therefore producing square channels. 
a)

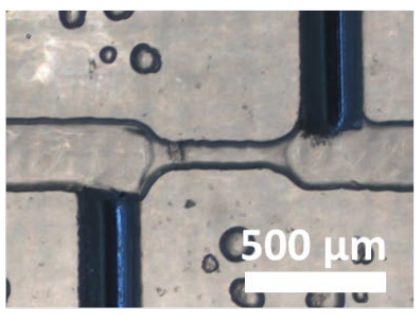

b)

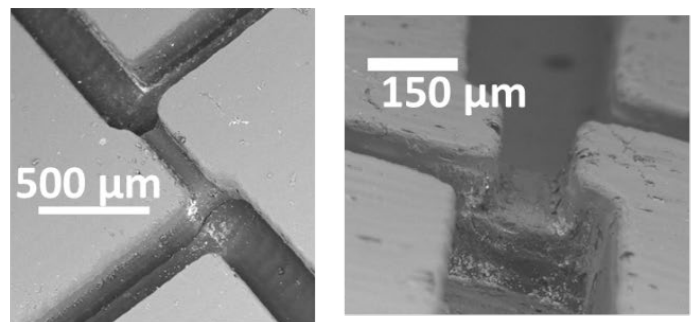

c)

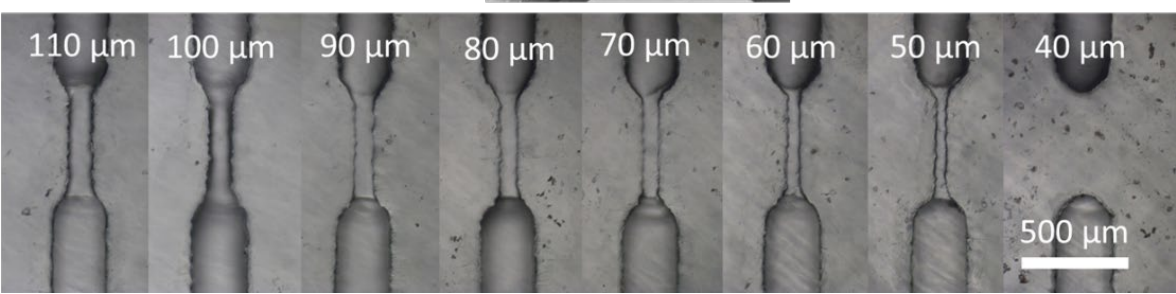

Figure 2a) Optical image of the sensing zone. b) SEM image of the flow channel, ramps and sensing zones. c) Optical images of the sensing zones from pores of different diameter.

Having tested the reproducibility of the AM process, prior to testing the RPS with particles, the electrochemical response was measured. Figure 3a shows the I-V response where $D_{s}$ and $I$ are 100 and $500 \mu \mathrm{m}$ respectively, equivalent graphs where I is equal to $100 \mu \mathrm{m}$ are shown in figure S3. Typical linear responses were observed, and the addition of a fluid flow through the pore did not alter the current response significantly, figure $3 \mathrm{~b}$. For the current amplifier used in this study the upper ionic strength of the solutions was $20 \mathrm{mM} \mathrm{KCl}$ as above this the baseline current quickly saturated the electronics even at low voltages. Figure $3 \mathrm{c}$ shows the measured base line current for typical conditions used here. The inset in figure $3 c$ shows the current response over twice the typical duration times of a particle during the RPS experiment. Figure $3 \mathrm{~d}$ shows the electric field lines originating from one and terminating at the other electrode. While the field lines are bent close to the electrodes due to their perpendicular orientation to the sensing channel they straighten out within the channel, indicating that the field is predominantly parallel to the channel walls and the electric potential declines in a linear fashion (compare figure S1). 

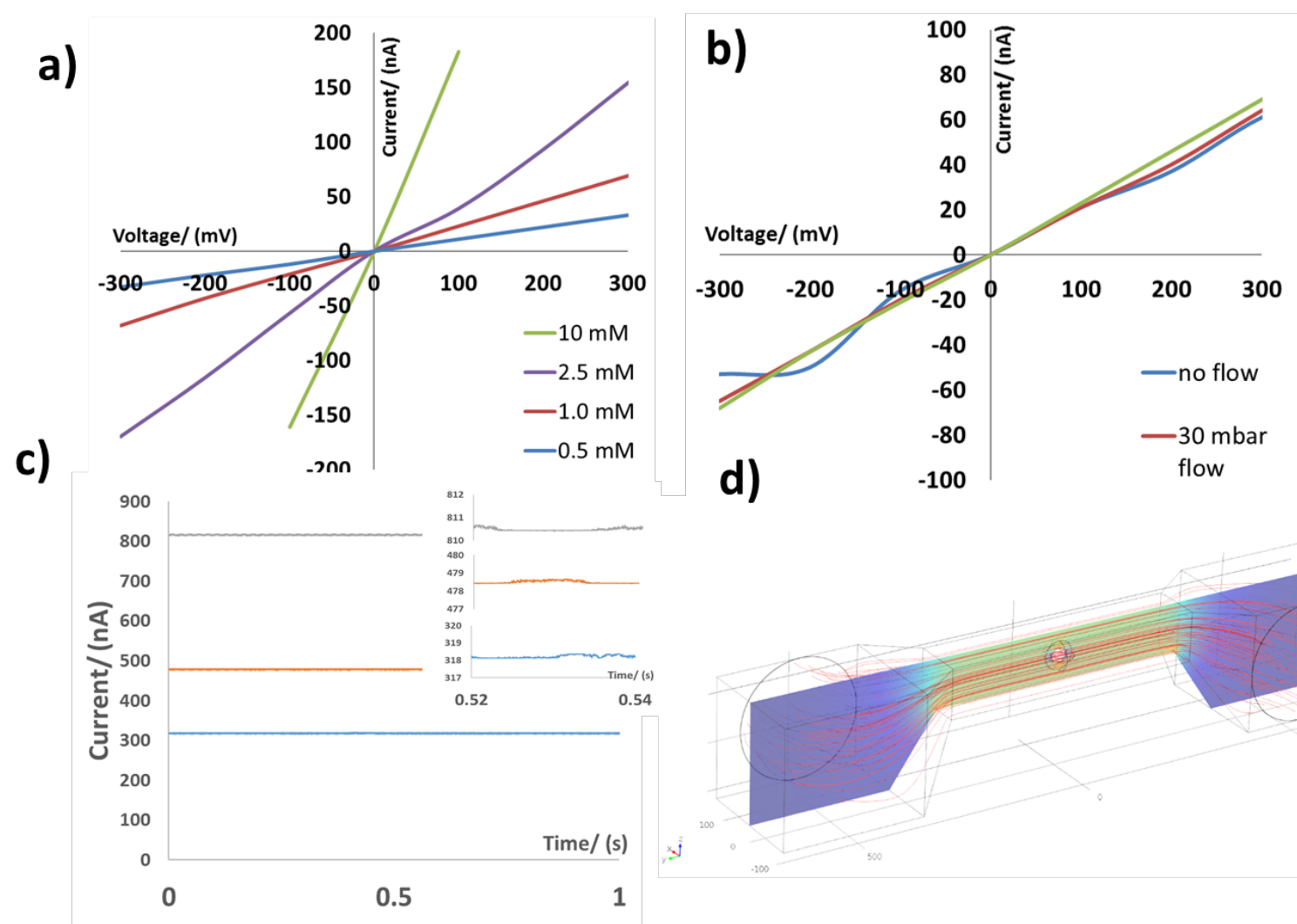

Figure 3a) Current voltage curves for when $D s=100 \mu \mathrm{m}$ and $\mathrm{I}=500 \mu \mathrm{m}$, at $50 \mathrm{mBar}$ flow rates, using $10 \mathrm{mM}$ (Green) $2.5 \mathrm{mM}$ (Purple) $1 \mathrm{mM}$ (Red) and $0.5 \mathrm{mM}$ (Blue) $\mathrm{KCl}$ solutions. b) Current voltage curves for the same chip as part a, filled with $1 \mathrm{mM} \mathrm{KCl} \mathrm{solution} \mathrm{under} \mathrm{different}$ flow rates, no flow (Blue), $30 \mathrm{mBar}$ flow (Red) and $50 \mathrm{mBar}$ flow (Green) c) Current time curves for the same chip described in part a. $0.25 \mathrm{mM} \mathrm{KCl}+4.5 \mathrm{~V}, 100 \mathrm{mbar}$ (Blue), $0.50 \mathrm{mM} \mathrm{KCl}$, +3.58V, 100 mbar (Orange), $5.0 \mathrm{mM} \mathrm{KCl},+1.3 \mathrm{~V}, 100 \mathrm{mbar}$ (Grey) d) 50 simulated electric field lines between the two electrodes (Ds and I are 105 and $545 \mu \mathrm{m}$ ).

To test the RPS sensors we added $30 \mu \mathrm{m}$ diameter particles to a solution that was passed through a channel with dimensions $D_{s}$ and I are 100 and $500 \mu \mathrm{m}$ respectively. The flow rates of the solution were varied using a pressure pump that ranged from 45 up to 105 mBar. Figure 4a shows how the flow rate has a small effect upon pulse magnitude (peak height), resulting in a small decrease in average pulse size from 1.1 to $0.89 \mathrm{nA}$ as the flow rate increases from 45 to $105 \mathrm{mBar}$. Figure $4 \mathrm{~b}$ illustrates the effect flow rate has on the baseline duration. As expected, when the flow rate was increased the duration decreases. This is due to the particles traveling with a greater velocity through the sensing zone, which at $105 \mathrm{mBar}$ was calculated to be $0.125 \mathrm{~m} / \mathrm{s}$. In all experiments the concentration of the particles remains constant and as the flow rate increases, the number of events per unit time also increases, figure 4c. Figure 4c demonstrates a linear relationship between applied pressure and pulse frequency. A scatter plot of blockade size versus duration from parts $a$ and $b$ is given in figure $4 \mathrm{~d}$. RPS devices offer a particle-by-particle analysis, each particle is analysed as it travels through the channel. The translocation of the particle produces a resistive pulse and examples of these recorded from the different flow rates, given in figure $4 \mathrm{e}$. It's interesting to note that rather than observe a pulse with a flat minimum that extents 
during the entire translocation, sharp decreases in current can be seen at the start and end of each pulse. The resistive pulse expected from a spherical particle with diameter $30 u m$ is shown in figure $4 \mathrm{f}$. The pulse shape reflects the geometry of the pore and the signal return quickly to the baseline outside the sensing region. While the baseline agrees reasonably well with the experiment (310 compared to $360 \mathrm{nA}$ ) given the uncertainty of the dimensions, the characteristic spikes of the pulses cannot be seen in the simulation
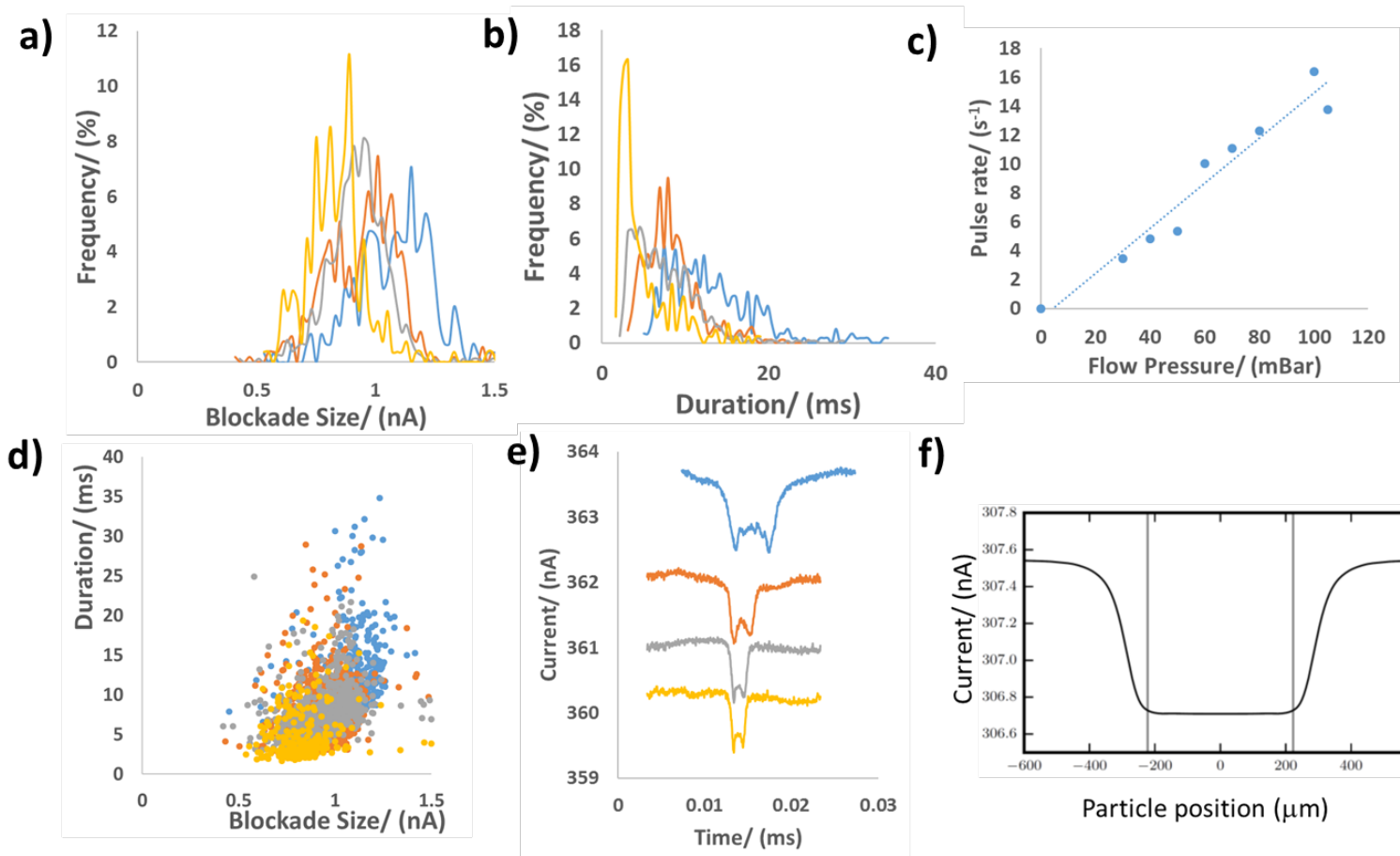

f)

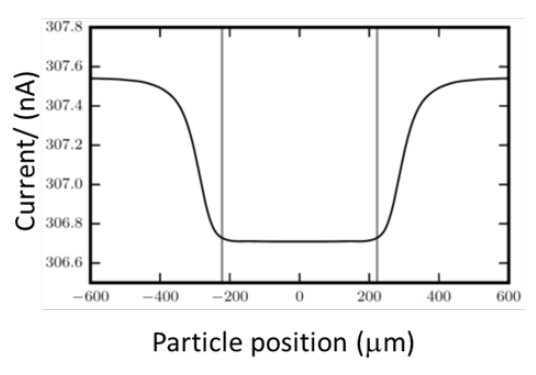

Figure 4. Data obtained using chip of Ds and I are 100 and $500 \mu \mathrm{m}$ respectively. $\mathrm{KCl}=0.25$ $m M$. Flow rates for the data are, Blue $=45$, Orange $=65$, Grey $=85$ and Yellow $105 \mathrm{mBar}$. Particle diameter $30 \mu \mathrm{m}$, at $7.5 \times 10^{3}$ particles $/ \mathrm{mL}$. Number of pulses recorded was 435,612 , 742 and 992 respectively. a) Distribution of pulse size for varying flow rates. b) Base line duration of events for differing flow rates. c) Average number of pulses per second recorded over a 1 min run. d) Scatter plot of blockade size versus duration. e) Example pulse shape for different flow rates. f) Predicted pulse shape based upon the FEM model (Ds = $105 \mu \mathrm{m}, I=$ $545 \mu \mathrm{m}, V=5.64 \mathrm{~V}, \sigma=3.675 \mathrm{mS} / \mathrm{m}(0.5 \mathrm{mM} \mathrm{KCl}$ concentration), Dparticle $=30 \mu \mathrm{m})$. The graph is centred around the middle of the sensing channel. The end of the sensing channel and the ramps are indicated by vertical solid and broken lines.

Irregular pulse shapes can be caused by a lack of uniformity within electric field, offaxial translocations, double layer interactions between the particles/ pore walls or irregularities within the dimensions of the channel themselves. ${ }^{10,45-51}$ The simulated electric field and potential are shown in figure $3 d$ and figure s1 respectively. Both show a uniform field and potential across the channel thus we excluded these as the cause of the current spikes seen on the edge of the pulses in figure $4 \mathrm{e}$. In this setup the size of the electric double layer around both the particle and pore wall would be insignificant, and unlikely to be the cause of the pulse shape. It was hypothesised that the shape of the pulse reflected the true shape of the channel i.e. each spike 
represents a change in channel dimension within the sensing zone. The decrease in current observed within these spikes indicates there is narrowing of the channel size, here the particle records different current blockages as it moves through the device. ${ }^{50}$ The optical and electron microscope images and analysis in figure 2 and S2 show smooth surfaces on the floor and walls of the RPS. Thus, we conclude that the likely source of the changing channel size would be the film placed on top. During the sealing stage some of the adhesive may collect along the pore walls or mouth. This is difficult to control within the current process and to ascertain its reproducibility a single device where $D_{s}$ and $/$ were 100 and $500 \mu \mathrm{m}$ was tested three times, between each data set the PC film was removed and replaced with a new layer. Figure S4a shows the distribution of pulse sizes for the same channel sealed on three different occasions. The variance from each RPS whilst present is low and the average pulse magnitudes for the three sensors were 1.002, 1.116 and $1.29 \mathrm{nA}$. The duration of the events for the three RPS sensors is very similar, 25.2, 20.79 and 22.78 shown in figure S4b. The data in figure S4 illustrates how the pulse size is more sensitive to changing the film, then the duration, example pulses are shown in figure S4c. Because a new PC film is used in each experiment, the shape and features of each pulse is unique to the setup. We attribute this to the sealing film forming a unique shape upon sealing in each experiment. As a final check to show that the pulse shape is related to the channel dimensions and not due to electric field or flow phenomenon two further experiment were carried out. The first was to record the particles flowing through the channel in one direction before then reversing the flow direction. The data in figure S5 shows how the pulses are mirror images of each other. Finally, we created a second smaller pore, with $D_{s}$ and $I$ at 80 and $500 \mu \mathrm{m}$ respectively. The same $30 \mu \mathrm{m}$ particles were passed through the channels at varying ionic strengths, from $0.25,0.5$ and $5 \mathrm{mM} \mathrm{KCl}$. Figure 5 shows an overlay of 10 pulses, with the average pulse shape overlaid in red. As can be seen from the data, increasing the ionic strength of the buffer had little effect on the pulse shape. It is also interesting to note that these pulses are different from the 100 $\mu \mathrm{m}$ diameter pore seen above. Again due to the fact that each pore is likely to have its own unique defects and gives rise to a unique pulse shape. 

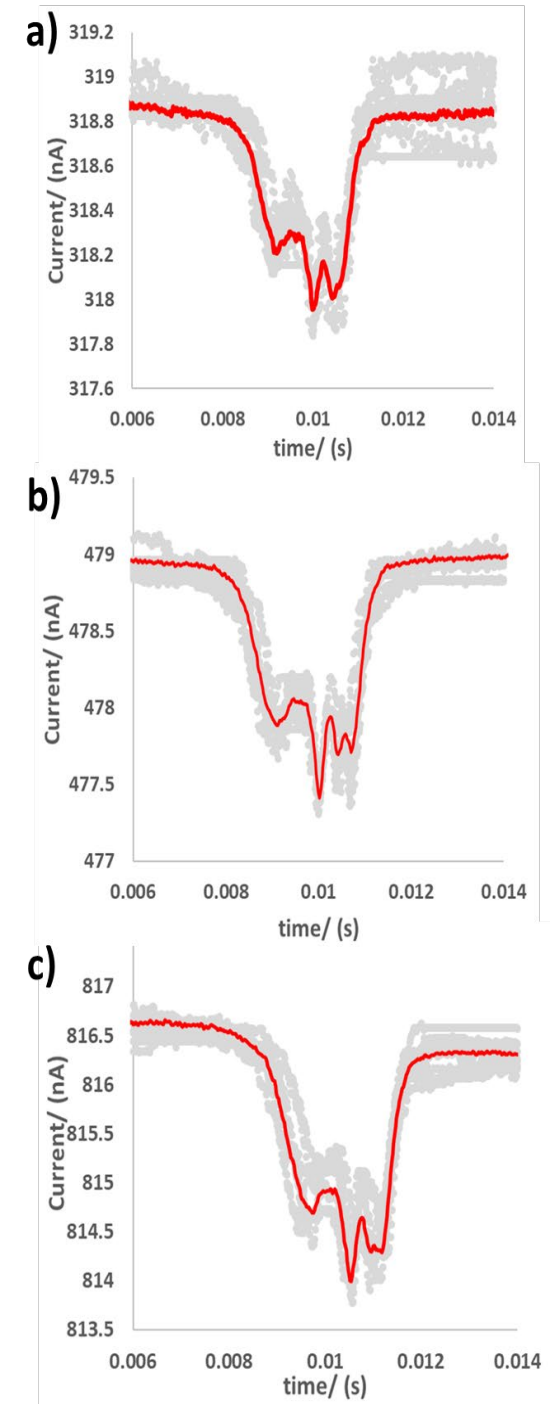

Figure 5 Overlap of 10 pulses and the average of all the data sets (red line) for channel Ds and $I$ are 80 and $500 \mu \mathrm{m}$ respectively. Flow rates are $100 \mathrm{mbar}$, particles diameter $30 \mu \mathrm{m}$, at $5 \times 10^{3}$ particles $/ \mathrm{mL}$ a) $0.25 \mathrm{mM} \mathrm{KCl}$, b) $0.5 \mathrm{mM} \mathrm{KCl}$, and c) $5 \mathrm{mM} \mathrm{KCl}$.

To further show the versatility of the AM process and illustrate how the channel dimensions and undulations effect the RPS signal, two constrictions were placed in series. This design has recently been used to characterise virus particles and nanomaterials, ${ }^{52}$ and have been shown to be powerful adaptation in RPS sensing. ${ }^{31,53}$ Figure 6 shows the AM design viewed through an optical microscope. They show a single and double RPS sensor. Figure 6c shows the RPS signal for the single constriction device, and a symmetrical pulse is observed, the relative pulse height was $0.00183 \pm 0.00007$ and duration $5.03 \pm 0.52 \mathrm{~ms}$. The signal for the dual RPS sensor shows that two pulses were observed. Analyses of the first pulse shows that the relative pulse height was $0.0017 \pm 0.00005$ and duration $2.06 \pm 0.36 \mathrm{~ms}$. The second pulse was $0.0016 \pm 0.00005 \mathrm{nA}$ and duration $2.4 \pm 0.36 \mathrm{~ms}$ with an average distance between the two pulses as $2.8 \pm 0.91 \mathrm{~ms}$. 

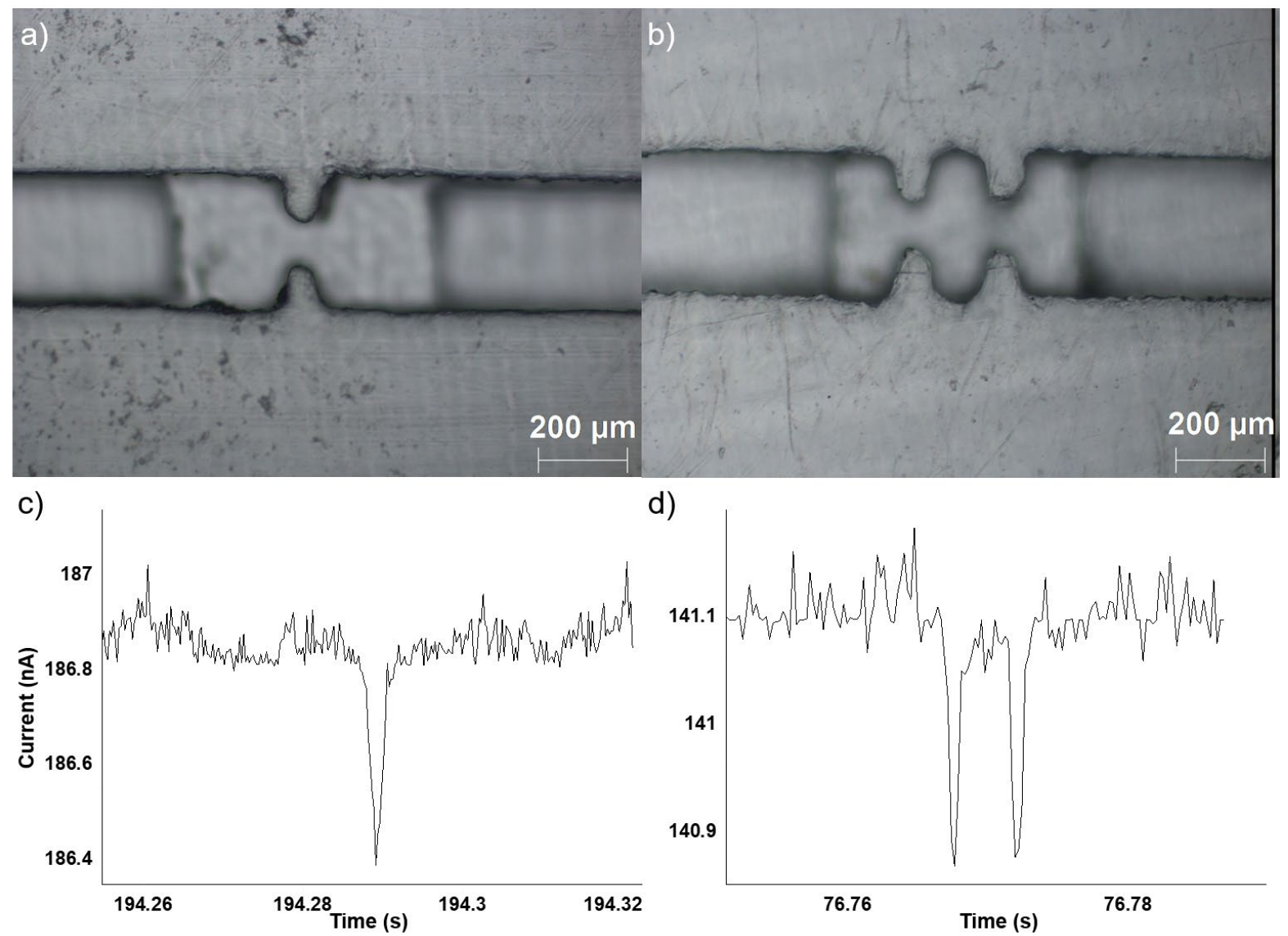

Figure 6 a) Microscope image of the one pore device which serves as a control, b) microscope image of the two-pore device as can be seen the pores are separated by a 100-micron gap, c) Pulse shape from the single constriction device d) Pulse shape from the two-constriction device, other examples are given in figure S6.

In RPS experiments the number of pulses per unit time can be used to determine the concentration of particles in solution. To use our devices for concentration analysis we wished to ascertain the variance of flow rates between different devices manufactured with the same dimensions, and the same device resealed on different occasions. We carried out a simple experiment of keeping the concentration of calibration particle constant and varying the flow rate. Figure s7 shows the relationship between flow rate and pulse frequency for multiple devices, and replicate runs on the same device sealed on multiple occasions. Similar to that observed in figure $4 \mathrm{c}$, a linear relationship between applied pressure and pulse frequency was recorded. The trend was observed across multiple devices and data showing the reproducibility of the particle rates for channels where I varies from 500 to $100 \mu \mathrm{m}$ are shown in figure $\mathrm{s} 7$.

Despite the irregular pulse shape from device to device, once sealed and operating the pulse shape remains consistent. The magnitude of the pulse is related to the volume of the particle traversing the sensing region. RPS devices have been used to determine the size, shape and zeta potential of samples. ${ }^{26,54,55}$ Where the pore dimensions are unknown calibration beads of a known size can be used, and as the pulse magnitude scales linearly with particle volume, a single point calibration allows 
the analysis of samples. Figure 7a shows how once calibrated the particle-by-particles analysis of the RPS sensor allows the simultaneous detection of 20 and $30 \mu \mathrm{m}$ particles. As mentioned above the number of pulses per unit time can also be used to determine the concentration of the particles. Figure $7 \mathrm{~b}$ shows a practical application of the relationship between pulse frequency and concentration. If the flow rate remains constant the pulse frequency is directly related to the particle concentration. By increasing the flow rate as illustrated in figure $4 \mathrm{c}$, the number of events observed increases. Thus, the limits of detection can be improved by increasing the flow rate.
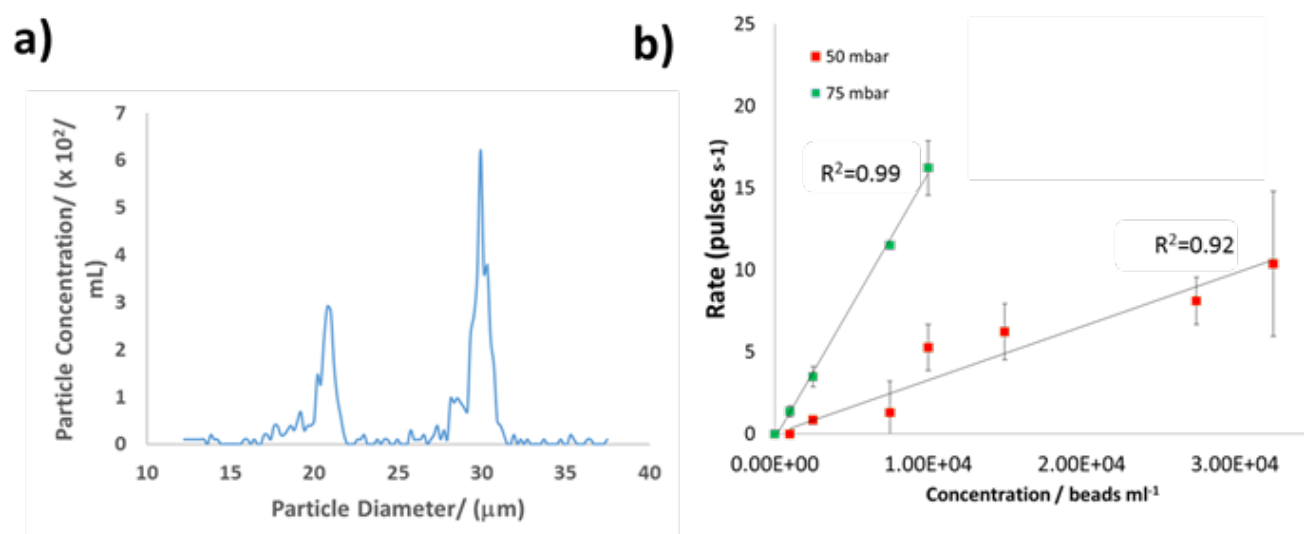

Figure 7 Data obtained using chip of Ds and I are 100 and $500 \mu m$ respectively a) Histogram of particle size versus concentration. b) Pulse frequency (rate) versus concentration,

\section{Conclusions}

We have successfully fabricated a resistive pulse sensor using additive manufacturing. The dimensions of the device are easily controlled, and the process allows for the channel dimension and length to the tailored. The devices are low cost and capable of being coupled directly into a flow device for the characterisation of material. Particle size, and concentration is monitored, and by controlled the flow rate concentrations as low as $1 \times 10^{-3}$ particles/ $\mathrm{mL}$ can be detected. The pulse shape was shown to be characteristic of the channel dimensions, and in future studies the analysis of the pulse may allow the channel shape, size and defects to be monitored without the need for microscopy.

\section{Acknowledgements}

The authors would like to thank Loughborough University for the funding of this research, and David Thompson in the Wolfson School of Mechanical, Electrical and Manufacturing Engineering for his support. The authors are grateful to the EPSRC Centre for Doctoral Training in Embedded Intelligence under grant reference EP/L014998/1 for financial support.

\section{Conflict of Interest}

The authors declare no conflict of interest 


\section{Supporting Information Available}

Supporting Information Available: The following files are available free of charge. Illustration of the design of the model, electric potential and field lines. Images of the RPS created with varying channel sizes, example pulses and signal versus flow rate for multiple devices. This material is available free of charge via the internet at http://pubs.acs.org

\section{References.}

(1) Dekker, C. Solid-State Nanopores. Nat Nano 2007, 2 (4), 209-215.

(2) Branton, D.; Deamer, D. W.; Marziali, A.; Bayley, H.; Benner, S. A.; Butler, T.; Di Ventra, M.; Garaj, S.; Hibbs, A.; Huang, X.; Jovanovich, S. B.; Krstic, P. S.; Lindsay, S.; Ling, X. S.; Mastrangelo, C. H.; Meller, A.; Oliver, J. S.; Pershin, Y. V.; Ramsey, J. M.; Riehn, R.; Soni, G. V.; Tabard-Cossa, V.; Wanunu, M.; Wiggin, M.; Schloss, J. A. The Potential and Challenges of Nanopore Sequencing. Nat Biotech 2008, 26 (10), 1146-1153.

(3) Sexton, L. T.; Horne, L. P.; Martin, C. R. Developing Synthetic Conical Nanopores for Biosensing Applications. Mol. Biosyst. 2007, 3 (10), 667-685.

(4) Sexton, L.; Horne, L.; Martin, C. Biosensing with Nanopores and Nanotubes. In Molecular- and Nano-Tubes SE - 6; Hayden, O., Nielsch, K., Eds.; Springer US, 2011; pp 165-207.

(5) Bayley, H.; Martin, C. R. Resistive-Pulse Sensing - From Microbes to Molecules. Chem. Rev. 2000, 100, 2575-2594.

(6) Bayley, H.; Cremer, P. S. Stochastic Sensors Inspired by Biology. Nature 2001, 413 (6852), 226-230.

(7) Kozak, D.; Anderson, W.; Vogel, R.; Trau, M. Advances in Resistive Pulse Sensors: Devices Bridging the Void between Molecular and Microscopic Detection. Nano Today 6 (5), 531-545.

(8) Roberts, G. S.; Yu, S.; Zeng, Q.; Chan, L. C. L.; Anderson, W.; Colby, A. H.; Grinstaff, M. W.; Reid, S.; Vogel, R. Tunable Pores for Measuring Concentrations of Synthetic and Biological Nanoparticle Dispersions. Biosens. Bioelectron. 2011, No. 0.

(9) Lan, W.-J.; Holden, D. A.; Liu, J.; White, H. S. Pressure-Driven Nanoparticle Transport across Glass Membranes Containing a Conical-Shaped Nanopore. J. Phys. Chem. C 2011, 115 (38), 18445-18452.

(10) Lan, W.-J.; Holden, D. A.; Zhang, B.; White, H. S. Nanoparticle Transport in Conical-Shaped Nanopores. Anal. Chem. 2011, 83 (10), 3840-3847.

(11) Whitehead, K. A.; Langer, R.; Anderson, D. G. Knocking down Barriers: Advances in SiRNA Delivery. Nat Rev Drug Discov 2009, 8, 129-138.

(12) Lee, S.; Zhang, Y.; White, H. S.; Harrell, C. C.; Martin, C. R. Electrophoretic Capture and Detection of Nanoparticles at the Opening of a Membrane Pore 
Using Scanning Electrochemical Microscopy. Anal. Chem. 2004, 76 (20), 6108-6115.

(13) Luo, L.; German, S. R.; Lan, W.-J.; Holden, D. a; Mega, T. L.; White, H. S. Resistive-Pulse Analysis of Nanoparticles. Annu. Rev. Anal. Chem. 2014, 7 (1), 513-535.

(14) Ali, M.; Ramirez, P.; Tahir, M. N.; Mafe, S.; Siwy, Z.; Neumann, R.; Tremel, W.; Ensinger, W. Biomolecular Conjugation inside Synthetic Polymer Nanopores Viaglycoprotein-Lectin Interactions. Nanoscale 2011, 3 (4), 1894-1903.

(15) Buchsbaum, S. F.; Nguyen, G.; Howorka, S.; Siwy, Z. S. DNA-Modified Polymer Pores Allow PH- and Voltage-Gated Control of Channel Flux. J. Am. Chem. Soc. 2014, 136 (28), 9902-9905.

(16) Yu, A. C. S.; Loo, J. F. C.; Yu, S.; Kong, S. K.; Chan, T. F. Monitoring Bacterial Growth Using Tunable Resistive Pulse Sensing with a Pore-Based Technique. Appl. Microbiol. Biotechnol. 2014, 98 (2), 855-862.

(17) Nam, S.; Choi, I.; Fu, C.; Kim, K.; Hong, S.; Choi, Y.; Zettl, A.; Lee, L. P. Graphene Nanopore with a Self-Integrated Optical Antenna. Nano Lett. 2014, 14 (10), 5584-5589.

(18) Henriquez, R. R.; Ito, T.; Sun, L.; Crooks, R. M. The Resurgance of Coulter Counting for Analysing Nanoscale Objects. Analyst 2004, 129, 478-482.

(19) Ito, T.; Sun, L.; Henriquez, R. R.; Crooks, R. M. A Carbon Nanotube-Based Coulter Nanoparticle Counter. Acc. Chem. Res. 2004, 37 (12), 937-945.

(20) Roberts, G. S.; Kozak, D.; Anderson, W.; Broom, M. F.; Vogel, R.; Trau, M. Tunable Nano/Micropores for Particle Detection and Discrimination: Scanning Ion Occlusion Spectroscopy. Small 2010, 6 (23), 2653-2658.

(21) Heng, J. B.; Aksimentiev, A.; Ho, C.; Marks, P.; Grinkova, Y. V; Sligar, S.; Schulten, K.; Timp, G. The Electromechanics of DNA in a Synthetic Nanopore. Biophys. J. 2006, 90 (3), 1098-1106.

(22) Lemmer, M.; Inkpen, M. S.; Kornysheva, K.; Long, N. J.; Albrecht, T. Unsupervised Vector-Based Classification of Single-Molecule Charge Transport Data. Nat. Commun. 2016, 7, 12922.

(23) Nguyen, G.; Howorka, S.; Siwy, Z. S. DNA Strands Attached Inside Single Conical Nanopores: Ionic Pore Characteristics and Insight into DNA Biophysics. J. Membr. Biol. 2011, 239 (1-2), 105-113.

(24) White, H. S.; Bund, A. Ion Current Rectification at Nanopores in Glass Membranes. Langmuir 2008, 24 (5), 2212-2218.

(25) Lan, W. J.; Kubeil, C.; Xiong, J. W.; Bund, A.; White, H. S. Effect of Surface Charge on the Resistive Pulse Waveshape during Particle Translocation through Glass Nanopores. J. Phys. Chem. C 2014, 118 (5), 2726-2734.

(26) Mayne, L.; Lin, C. Y.; Christie, S. D. R.; Siwy, Z. S.; Platt, M. The Design and Characterization of Multifunctional Aptamer Nanopore Sensors. ACS Nano 2018, 12 (5), 4844-4852. 
(27) Vogel, R.; Willmott, G.; Kozak, D.; Roberts, G. S.; Anderson, W.; Groenewegen, L.; Glossop, B.; Barnett, A.; Turner, A.; Trau, M. Quantitative Sizing of Nano/Microparticles with a Tunable Elastomeric Pore Sensor. Anal. Chem. 2011, 83 (9), 3499-3506.

(28) Blundell, E. L. C. J.; Mayne, L. J.; Billinge, E. R.; Platt, M. Emergence of Tunable Resistive Pulse Sensing as a Biosensor. Anal. Methods 2015.

(29) Peng, R.; Li, D. Particle Detection on Microfluidic Chips by Differential Resistive Pulse Sensing (RPS) Method. Talanta 2018, 184, 418-428.

(30) Yasaki, H.; Yasui, T.; Yanagida, T.; Kaji, N.; Kanai, M.; Nagashima, K.; Kawai, T.; Baba, Y. Substantial Expansion of Detectable Size Range in Ionic Current Sensing through Pores by Using a Microfluidic Bridge Circuit. J. Am. Chem. Soc. 2017, 139 (40), 14137-14142.

(31) Hinkle, P.; Westerhof, T. M.; Qiu, Y.; Mallin, D. J.; Wallace, M. L.; Nelson, E. L.; Taborek, P.; Siwy, Z. S. A Hybrid Resistive Pulse-Optical Detection Platform for Microfluidic Experiments. Sci. Rep. 2017, 7 (1), 10173.

(32) Hampson, S. M.; Rowe, W.; Christie, S. D. R.; Platt, M. 3D Printed Microfluidic Device with Integrated Optical Sensing for Particle Analysis. Sensors Actuators B Chem. 2018, 256 (Supplement C), 1030-1037.

(33) Capel, A. J.; Wright, A.; Harding, M. J.; Weaver, G. W.; Li, Y.; Harris, R. A.; Edmondson, S.; Goodridge, R. D.; Christie, S. D. R. 3D Printed Fluidics with Embedded Analytic Functionality for Automated Reaction Optimisation. Beilstein J. Org. Chem. 2017, 13, 111-119.

(34) Monaghan, T.; Harding, M. J.; Harris, R. A.; Friel, R. J.; Christie, S. D. R. Customisable 3D Printed Microfluidics for Integrated Analysis and Optimisation. Lab Chip 2016, 16 (17), 3362-3373.

(35) Okafor, O.; Weilhard, A.; Fernandes, J. A.; Karjalainen, E.; Goodridge, R.; Sans, V. Advanced Reactor Engineering with 3D Printing for the ContinuousFlow Synthesis of Silver Nanoparticles. React. Chem. Eng. 2017.

(36) Capel, A. J.; Rimington, R. P.; Lewis, M. P.; Christie, S. D. R. 3D Printing for Chemical, Pharmaceutical and Biological Applications. Nat. Rev. Chem. 2018, 2 (12), 422-436.

(37) Bhattacharjee, N.; Urrios, A.; Kang, S.; Folch, A. The Upcoming 3D-Printing Revolution in Microfluidics. Lab Chip 2016, 16 (10), 1720-1742.

(38) Bayley, H.; Martin, C. R. Resistive-Pulse SensingFrom Microbes to Molecules. Chem. Rev. 2000, 100 (7), 2575-2594.

(39) Menestrina, J.; Yang, C.; Schiel, M.; Vlassiouk, I.; Siwy, Z. S. Charged Particles Modulate Local Ionic Concentrations and Cause Formation of Positive Peaks in Resistive-Pulse-Based Detection. J. Phys. Chem. C 2014, 118 (5), 2391-2398.

(40) DeBlois, R. W.; Bean, C. P. Counting and Sizing of Submicron Particles by the Resistive Pulse Technique. Rev. Sci. Instrum. 1970, 41 (7), 909-916.

(41) Gregg, E. C.; Steidley, K. D. Electrical Counting and Sizing of Mammalian 
Cells in Suspension. Biophys. J. 1965, 5 (4), 393-405.

(42) McgrawHill. An Introduction to the Finite Element Method; 1993.

(43) German, S. R.; Luo, L.; White, H. S.; Mega, T. L. Controlling Nanoparticle Dynamics in Conical Nanopores. J. Phys. Chem. C 2013, 117 (1), 703-711.

(44) Wang, Z. Q. and J. Z. and G.-X. Effects of Particle's off-Axis Position, Shape, Orientation and Entry Position on Resistance Changes of Micro Coulter Counting Devices. Meas. Sci. Technol. 2011, 22 (4), 45804.

(45) Menestrina, J.; Yang, C.; Schiel, M.; Vlassiouk, I.; Siwy, Z. S. Charged Particles Modulate Local lonic Concentrations and Cause Formation of Positive Peaks in Resistive-Pulse-Based Detection. J. Phys. Chem. C 2014, 118 (5), 2391-2398.

(46) Siwy, Z. S. Ion-Current Rectification in Nanopores and Nanotubes with Broken Symmetry. Adv. Funct. Mater. 2006, 16 (6), 735-746.

(47) Weatherall, E.; Willmott, G. R. Conductive and Biphasic Pulses in Tunable Resistive Pulse Sensing. J. Phys. Chem. B 2015, 119 (16), 5328-5335.

(48) Storm, A. J.; Storm, C.; Chen, J.; Zandbergen, H.; Joanny, J. F.; Dekker, C. Fast DNA Translocation through a Solid-State Nanopore. Nano Lett. 2005, 5 (7), 1193-1197.

(49) Hsu, W. L.; Daiguji, H. Manipulation of Protein Translocation through Nanopores by Flow Field Control and Application to Nanopore Sensors. Anal. Chem. 2016, 88 (18), 9251-9258.

(50) Pevarnik, M.; Healy, K.; Toimil-Molares, M. E.; Morrison, A.; Létant, S. E.; Siwy, Z. S. Polystyrene Particles Reveal Pore Substructure As They Translocate. ACS Nano 2012, 6 (8), 7295-7302.

(51) Balakrishnan, K. R.; Whang, J. C.; Hwang, R.; Hack, J. H.; Godley, L. A.; Sohn, L. L. Node-Pore Sensing Enables Label-Free Surface-Marker Profiling of Single Cells. Anal. Chem. 2015, 87 (5), 2988-2995.

(52) Zhou, J.; Kondylis, P.; Haywood, D. G.; Harms, Z. D.; Lee, L. S.; Zlotnick, A.; Jacobson, S. C. Characterization of Virus Capsids and Their Assembly Intermediates by Multicycle Resistive-Pulse Sensing with Four Pores in Series. Anal. Chem. 2018, 90 (12), 7267-7274.

(53) Harms, Z. D.; Mogensen, K. B.; Nunes, P. S.; Zhou, K.; Hildenbrand, B. W.; Mitra, I.; Tan, Z.; Zlotnick, A.; Kutter, J. P.; Jacobson, S. C. Nanofluidic Devices with Two Pores in Series for Resistive-Pulse Sensing of Single Virus Capsids. Anal. Chem. 2011, 83 (24), 9573-9578.

(54) Blundell, E. L. C. J.; Vogel, R.; Platt, M. Particle-by-Particle Charge Analysis of DNA-Modified Nanoparticles Using Tunable Resistive Pulse Sensing. Langmuir 2016, 32 (4).

(55) Mayne, L. J.; Christie, S. D. R.; Platt, M. A Tunable Nanopore Sensor for the Detection of Metal lons Using Translocation Velocity and Biphasic Pulses. Nanoscale 2016, 8 (45), 19139-19147. 
For TOC only

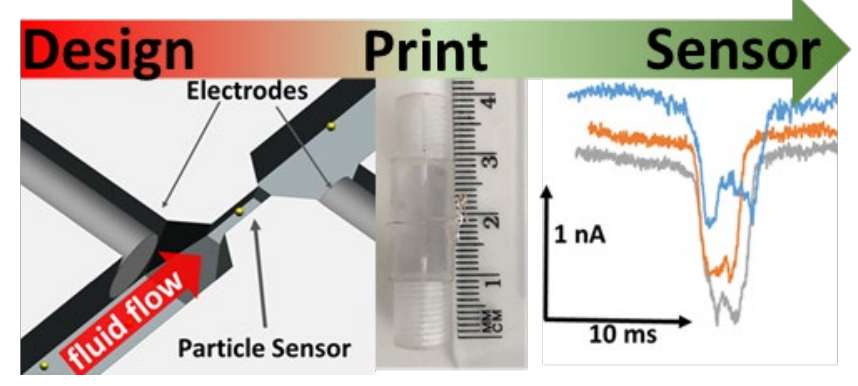

